\section{Lichen Scrofulosorum}

A 9-year-old boy presented with asymptomatic, skin colored, grouped papules with mild scaling on elbows, buttocks, knees, and dorsa of feet in a bilateral symmetric manner for 8 months (Fig. 1). There were no systemic features and boy appeared otherwise healthy. Family history was non-contributory. Mantoux text was positive (20 X $24 \mathrm{~mm}$ ); routine blood investigations, chext $X$-ray and abdominal ultrasonography were normal. Histopathological findings were consistent with lichen scrofulosorum (Fig. 2). He showed almost complete resolution of lesions after four months of anti-tubercular therapy.

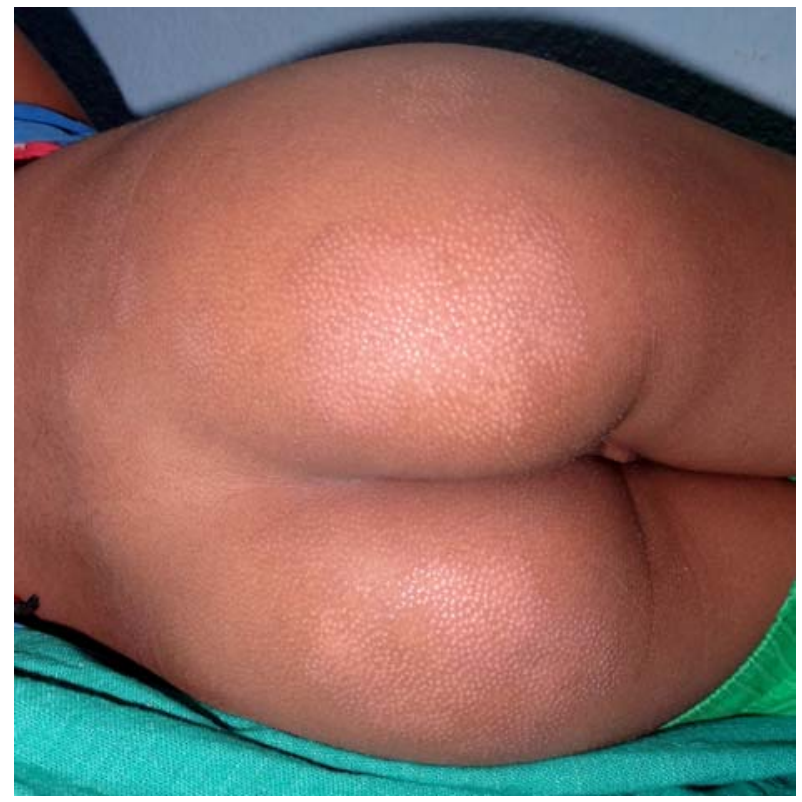

FIG. 1 Symmetric skin colored, grouped, papules with mild scaling on buttocks.

Lichen scrofulosorum, an uncommon tuberculid, is usually seen in children with nodal or skeletal tuberculosis or following BCG vaccination. Lesions are usually

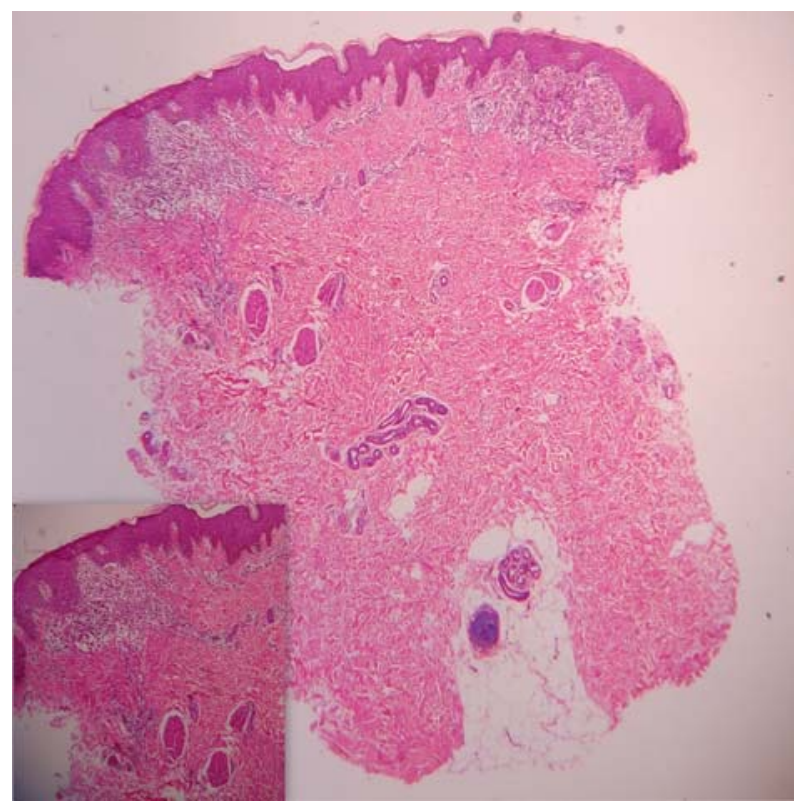

Fig. 2 Irregular acanthosis and inflammatory cells in upper dermis (H\&E X40); Inset: non-caseating granuloma in upper dermis (H\&E X100).

confined to the trunk and present as asymptomatic, firm, follicular or perifollicular flat-topped skin-colored or reddish brown papules, sometimes with fine scales. Lesions may coalesce to form rough, discoid plaques, and may persist for months. With anti-tubercular treatment, the lesions usually clear within 12 weeks without scarring. The differential diagnoses include lichen nitidus (more shiny), lichen spinulosus, keratosis pilaris (keratotic projections- antenna sign), phrynoderma, secondary syphilis, papular sarcoidosis, pityriasis rubra pilaris, and folliculitis. Histopathology is diagnostic and demonstrates superficial granulomas around hair follicles and sweat ducts, with little or no caseation necrosis.

Piyush Kumar, OP Jha ANd *AviJit Mondal Departments of Dermatology, Katihar Medical College, Bihar and *College of Medicine and JNM Hospital, Kalyani, West Bengal, India. docpiyush@gmail.com 